\title{
Learning and Classification of Malware Behavior
}

Konrad Rieck ${ }^{1}$, Thorsten Holz², Carsten Willems², Patrick Düssel ${ }^{1}$, and Pavel Laskov ${ }^{1}$

DIMVA 2008, Paris, France

1 Fraunhofer Institute FIRST, Germany

2 University of Mannheim, Germany 


\section{Malware today}

- Malicious software: A vivid threat

- Plethora of worms, trojans, bots, backdoors

- Exponential growth of malware in the wild

- Emergence of criminal "industries"

- Conventional static defenses insufficient

- High degree of polymorphy and obfuscation
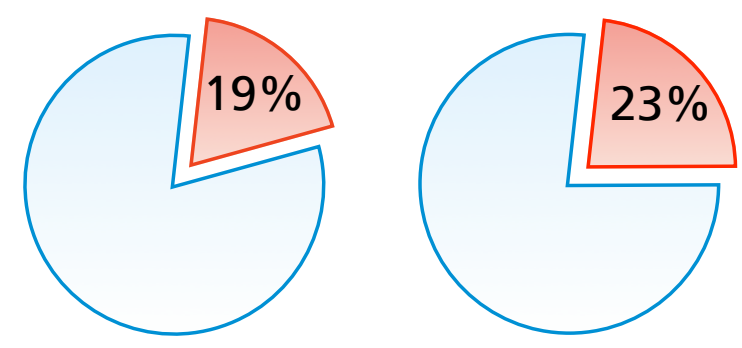

BitDefender

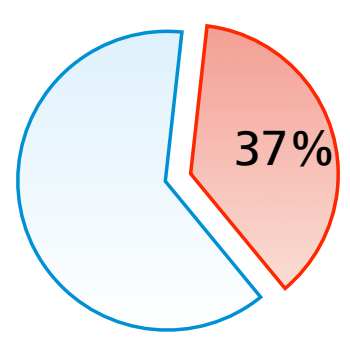

ClamAV
Undetected

Anti-virus producs

Evaluation on 4,000 malware binaries 


\section{Dynamic analysis}

- Malware behavior

- Malware differs in purpose and functionality

- Typical and discriminative behavioral patterns

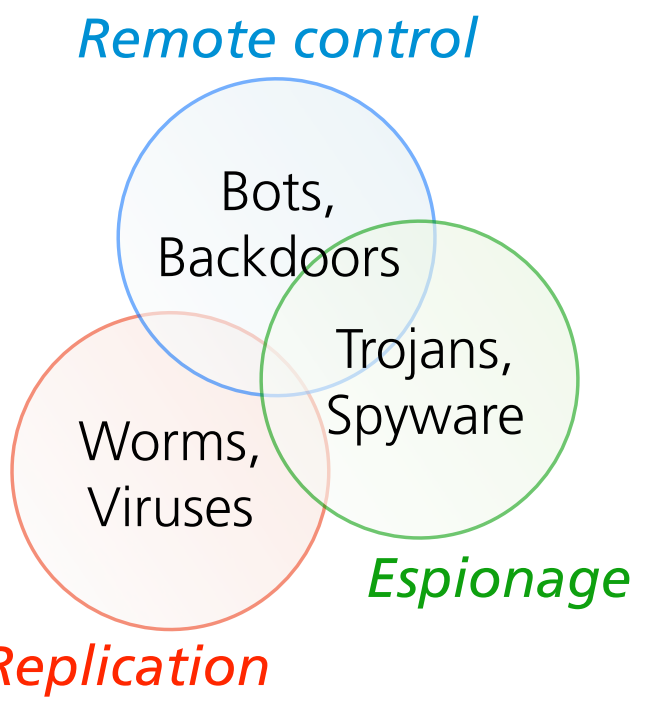

- Behavior-based analysis

- Monitoring and detection of malicious behavior

- AV products: manually generated behavior rules

- Alternative, fully automated approaches? 


\section{Learning-based approaches}

- Clustering of malware behavior (e.g. Bailey et al., RAID 2007)

- Difficult to control cluster models (many vs. few)

- Clustering often non-predictive, e.g. linkage clustering

- Idea: Generalize from behavior and prior knowledge

- Incorporate (noisy) labels, e.g. by anti-virus tool

- Learn classification of malware families using labels

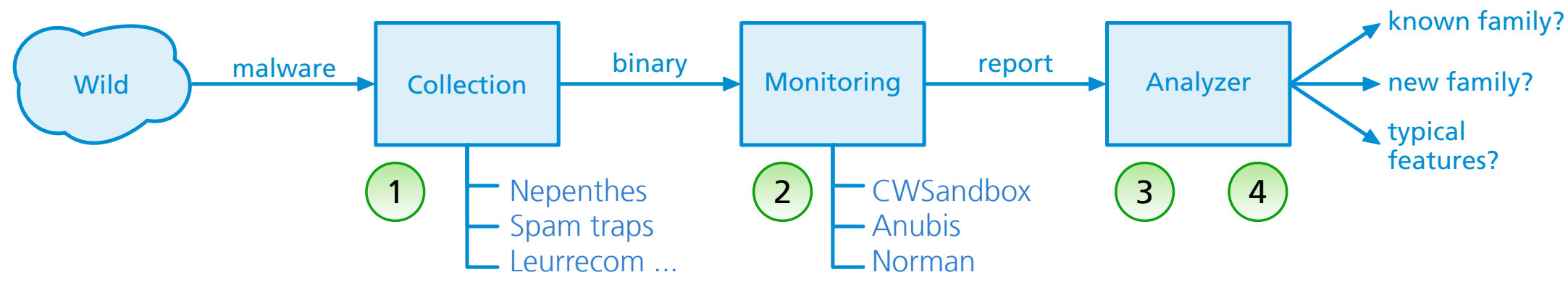




\section{Collecting malware}

- Automatic collection of current malware families

- Broad range of malware using diverse methods, e.g. honeypots, spam traps, honeyclients

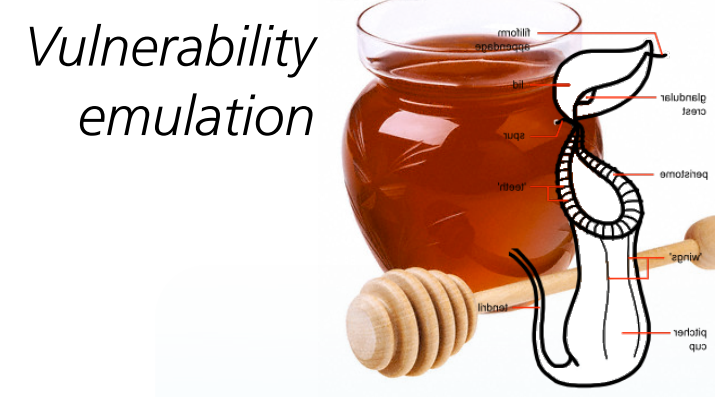

Nepenthes

Self-replicating malware

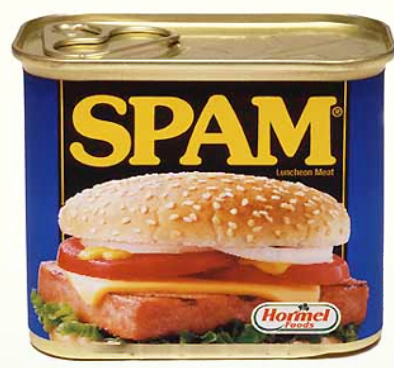

Spam traps

Trojans and backdoors

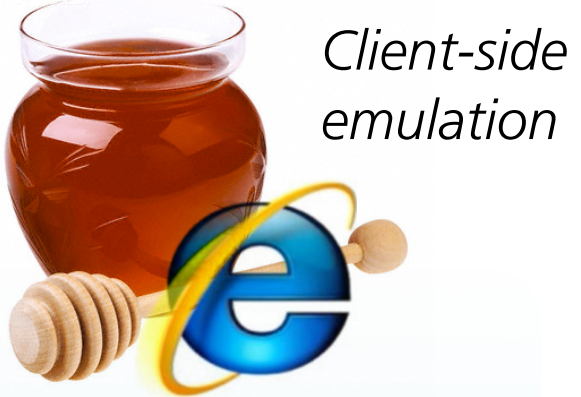

Honeyclients

Drive-by malware 


\section{Monitoring malware}

- Sandbox for malware

- Protected execution environment (e.g. CWSandbox)

- Monitors and reports observed behavior

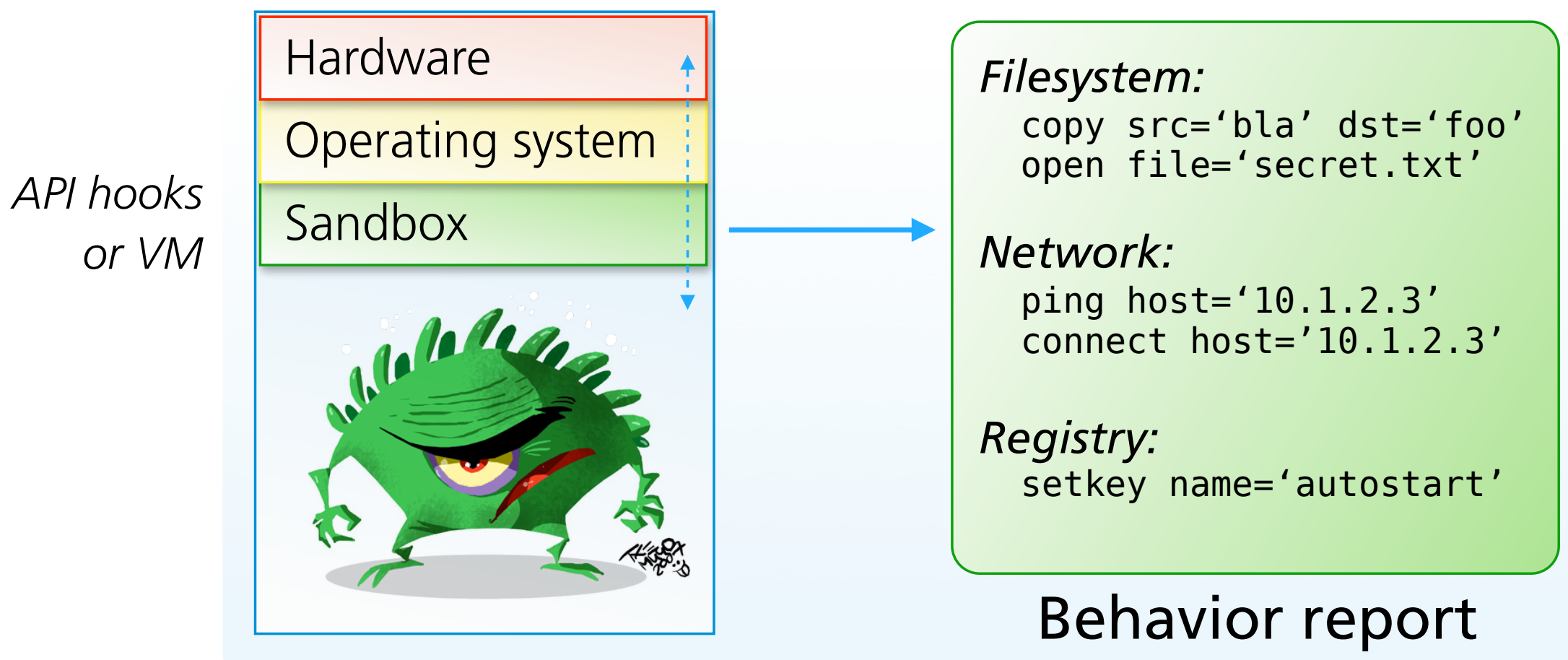




\section{Feature extraction 3}

Report $x$

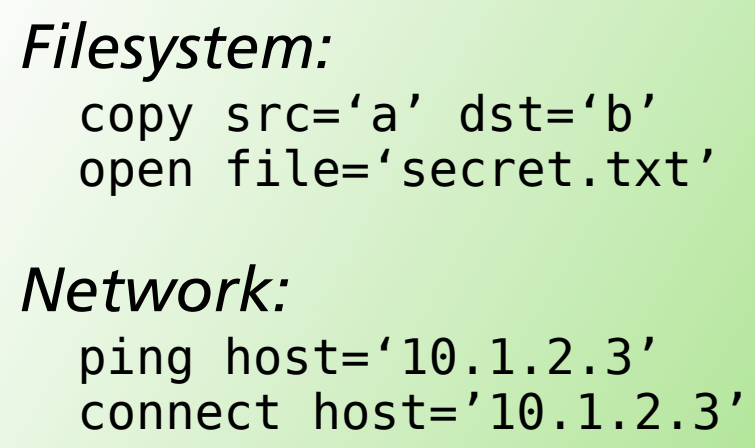

Extraction
Feature vector

$$
\left.\begin{array}{l}
\longrightarrow \\
\longrightarrow \text { copy src='a' dst='b' }
\end{array} \quad \begin{array}{c}
f\left(x, s_{1}\right) \\
f\left(x, s_{2}\right) \\
\vdots
\end{array}\right)
$$




\section{Learning behavior (4)}

- Discrimination of malware families in feature space

- Assign family label to embedded reports, e.g. AV label

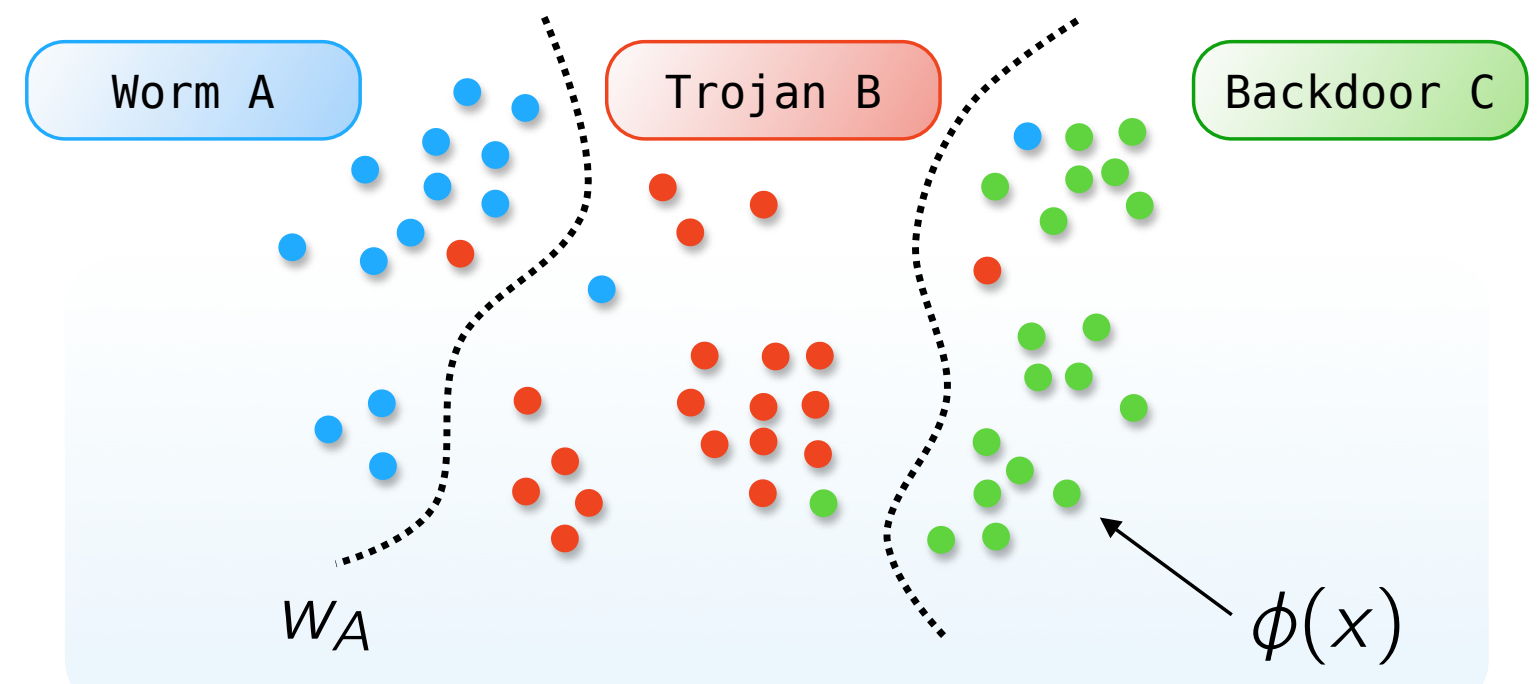

- Learn maximum-margin hyperplane $w$ for each family

- Incorporation of non-linearity using kernel functions 


\section{Experimental evaluation}

- Malware collection labeled using AV tool (AntiVir)

- \#k: 10,072 malware binaries from 14 families

- \#u: 3,139 unknown variants (detected 4 weeks later)

\begin{tabular}{lrr|crr} 
Malware family & \#k & \#u & Malware family & \#k & $\#$ u \\
\hline 1: Backdoor.VanBot & 91 & 169 & 8: Worm.Korgo & 244 & 4 \\
\hline 2: Trojan.Bancos & 279 & 208 & 9: Worm.Parite & 1215 & 19 \\
\hline 3: Trojan. Banker & 834 & 185 & 10: Worm.PoeBot & 140 & 188 \\
\hline 4: Worm.Allaple & 1500 & 614 & 11: Worm.Rbot & 1399 & 904 \\
\hline 5: Worm.Doomber & 426 & 0 & 12: Worm.Sality & 661 & 0 \\
\hline 6: Worm.Gobot & 777 & 0 & 13: Worm.SdBot & 777 & 597 \\
\hline 7: Worm.IRCBot & 229 & 107 & 14: Worm.Virut & 1500 & 144 \\
\hline
\end{tabular}




\section{Results: Classification}

- Learning on known, prediction on unknown variants

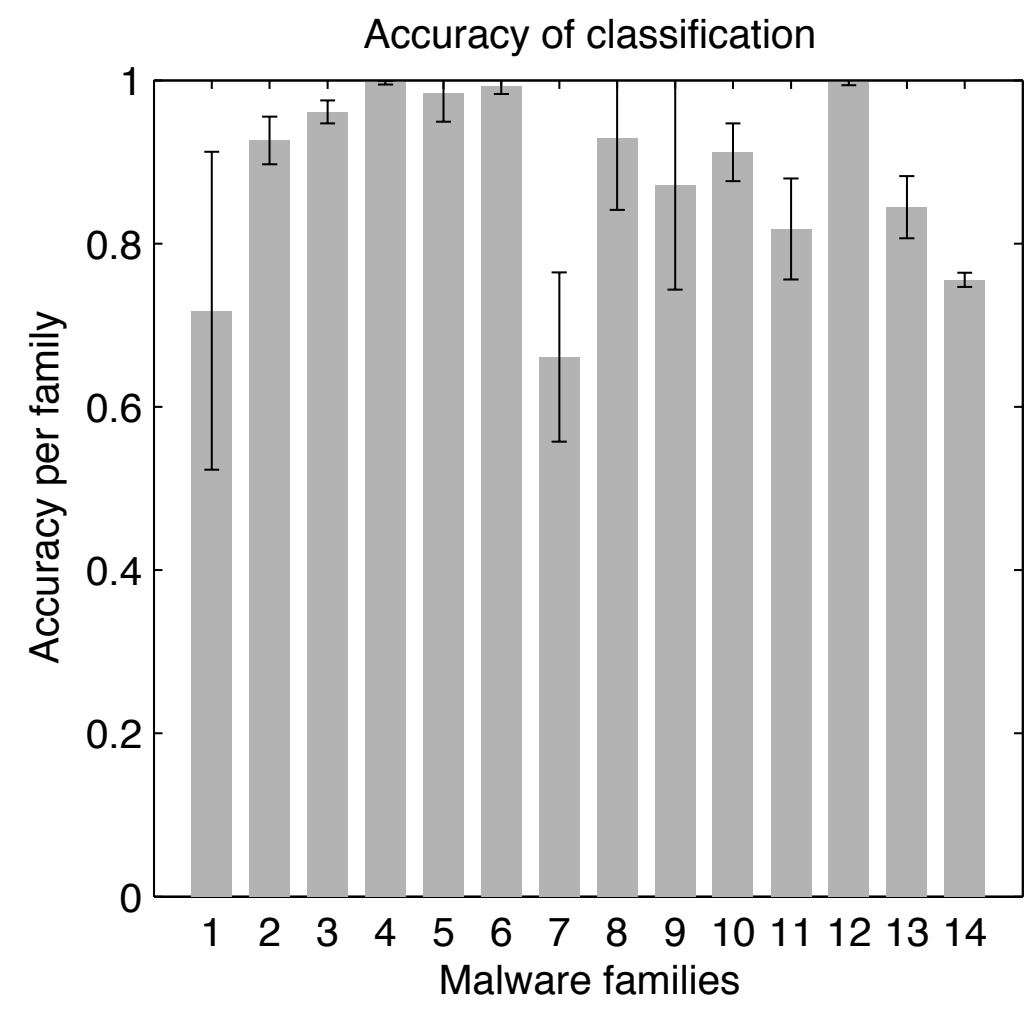

Known variants, avg. 88\%

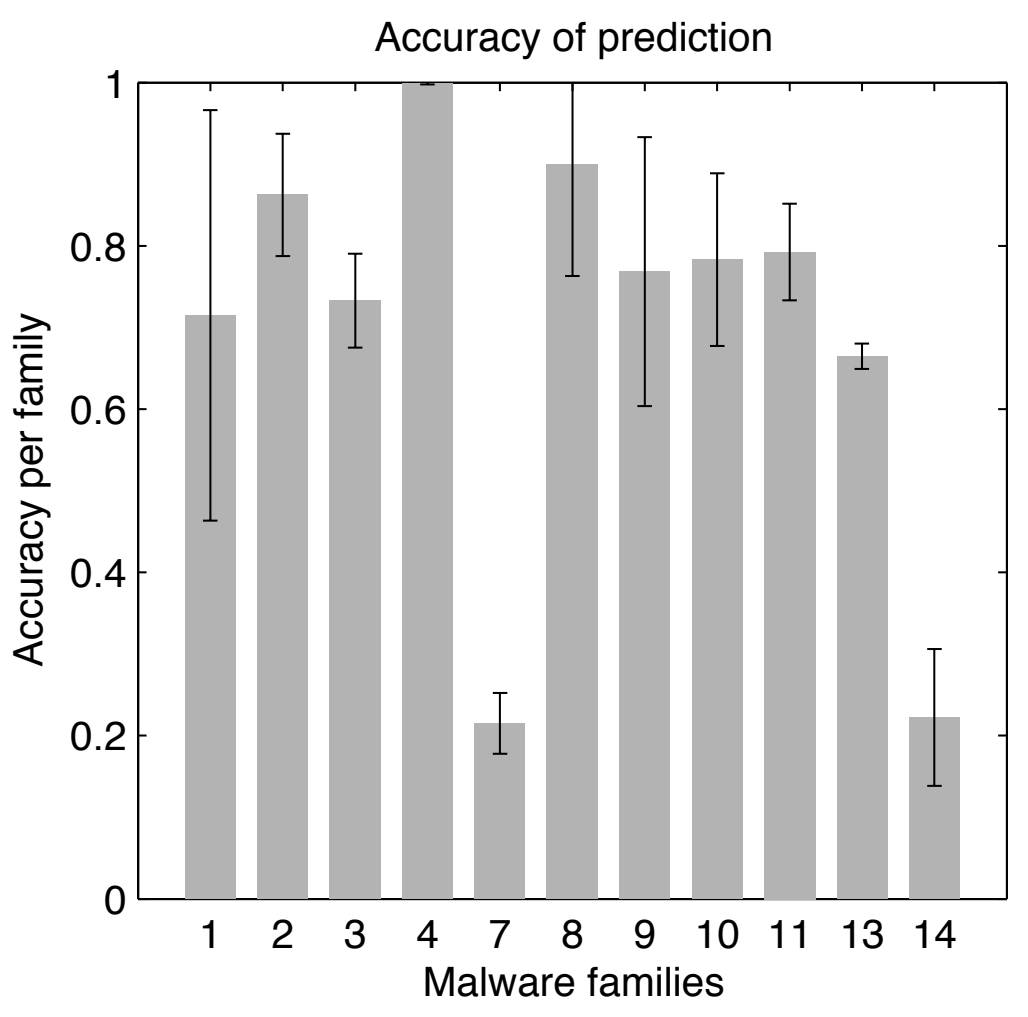

Unknown variants, avg. $69 \%$

- High detection accuracy (Note: random guessing $=7 \%$ ) 
- Explanation of learned malware behavior classifier

- Most discriminative dimensions in hyperplane vectors

\section{Worm.Sality}

\begin{tabular}{|c|c|}
\hline $\begin{array}{l}0.0142: \\
0.0073: \\
0.0068: \\
0.0051: \\
0.0035:\end{array}$ & 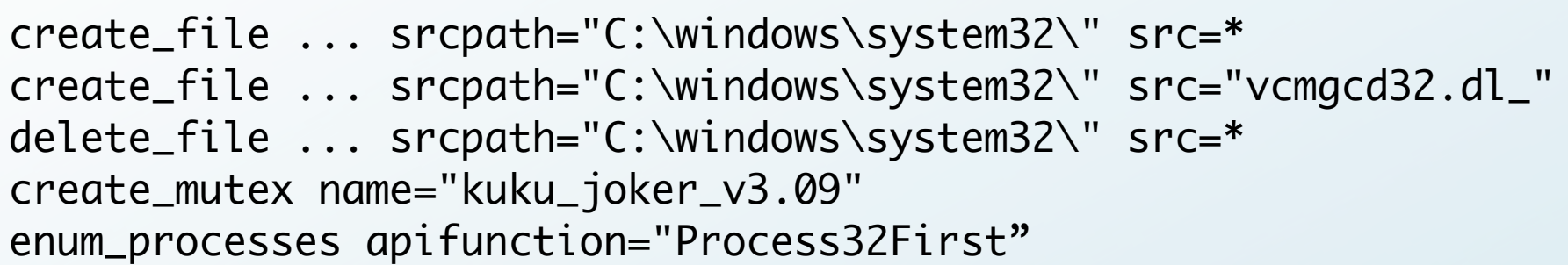 \\
\hline
\end{tabular}

\section{Worm.Doomber}

\begin{tabular}{l|l|}
$0.0084:$ & create_mutex name="GhostBOT0.58c" \\
$0.0073:$ & create_mutex name="GhostBOT0.58b" \\
$0.0052:$ & create_mutex name="GhostBOT0.58a" \\
$0.0014:$ & enum_processes apifunction="Process32First" \\
$0.0011:$ & query_value key="HKEY_LOCAL_MACHINE $\backslash . .$. . $r u n "$ value="GUARD" \\
\hline
\end{tabular}


- Behavior-based malware analysis

- Extension of current AV tools (see Oberheide et al., USENIX 2008)

- Hinders simple obfuscation and polymorphy

- Supervised learning on malware behavior

- Detection accuracy: 69\% unknown malware variants

- No black box: Explanation via hyperplane vectors

- Further extension: Rejection of unknown behavior

- Perspectives

- Semi-supervised learning: Best of both worlds. 
Thanks. Questions? 
- Evasion attacks

- Detection of honeypot or sandbox environment

- Obfuscated and polymorphic behavior

- Mimic behavior of benign programs or other malware

- Consequences \& defenses

- Run multiple honeypots and sandboxes in parallel

- Obfuscation and polymorphy: Discriminative features?

- Fruitless to mimic benign program $=$ No real activity 


\section{References}

- Bächer, Kötter, Holz, Dornseif, Freiling. The Nepenthes platform: An efficient approach to collect malware. RAID 2006.

- Bailey, Oberheide, Andersen, Mao, Jahanian, Nazario. Automated classification and analysis of Internet malware. RAID 2007.

- Burges. A Tutorial on Support Vector Machines for Pattern Recognition. Knowledge Discovery and Data Mining 2(2), 1998.

- Oberheide, Cooke, Jahanian. N-Version Antivirus in the Network Cloud. USENIX 2008.

- Rieck, Laskov. Linear-Time Computation of Similarity Measure for Sequential Data. Journal of Machine Learning Research 9(1), 2008.

- Willems, Holz, Freiling. Towards automated dynamic binary analysis, IEEE Magazine Security \& Privacy 5(2), 2007. 


\section{Unsupervised vs. Supervised}

- Clustering (unsupervised)

- Determine malware families from structure only

- Difficult to control model complexity without labels

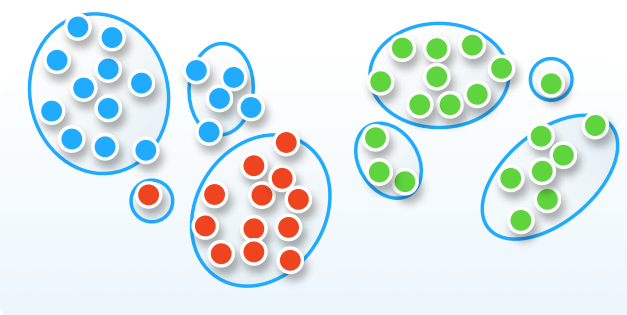

- Classification (supervised)

- Determine malware families using structure and labels

- Generalization beyond noisy labels

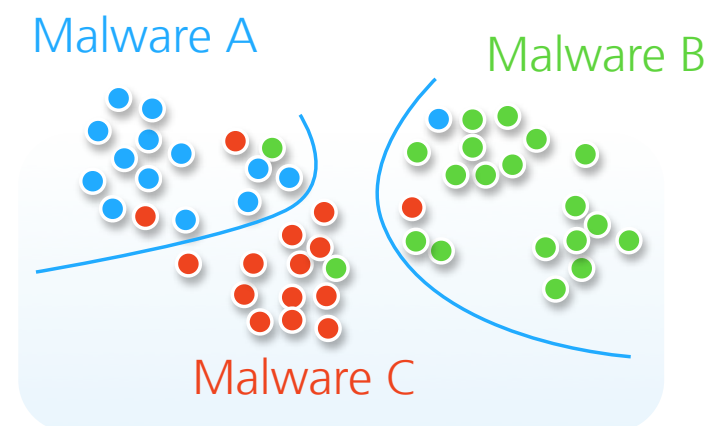




\section{Unknown behavior}

- Rejection of unknown behavior

- Probabilistic fit on output of classifier (reject if <0.5)
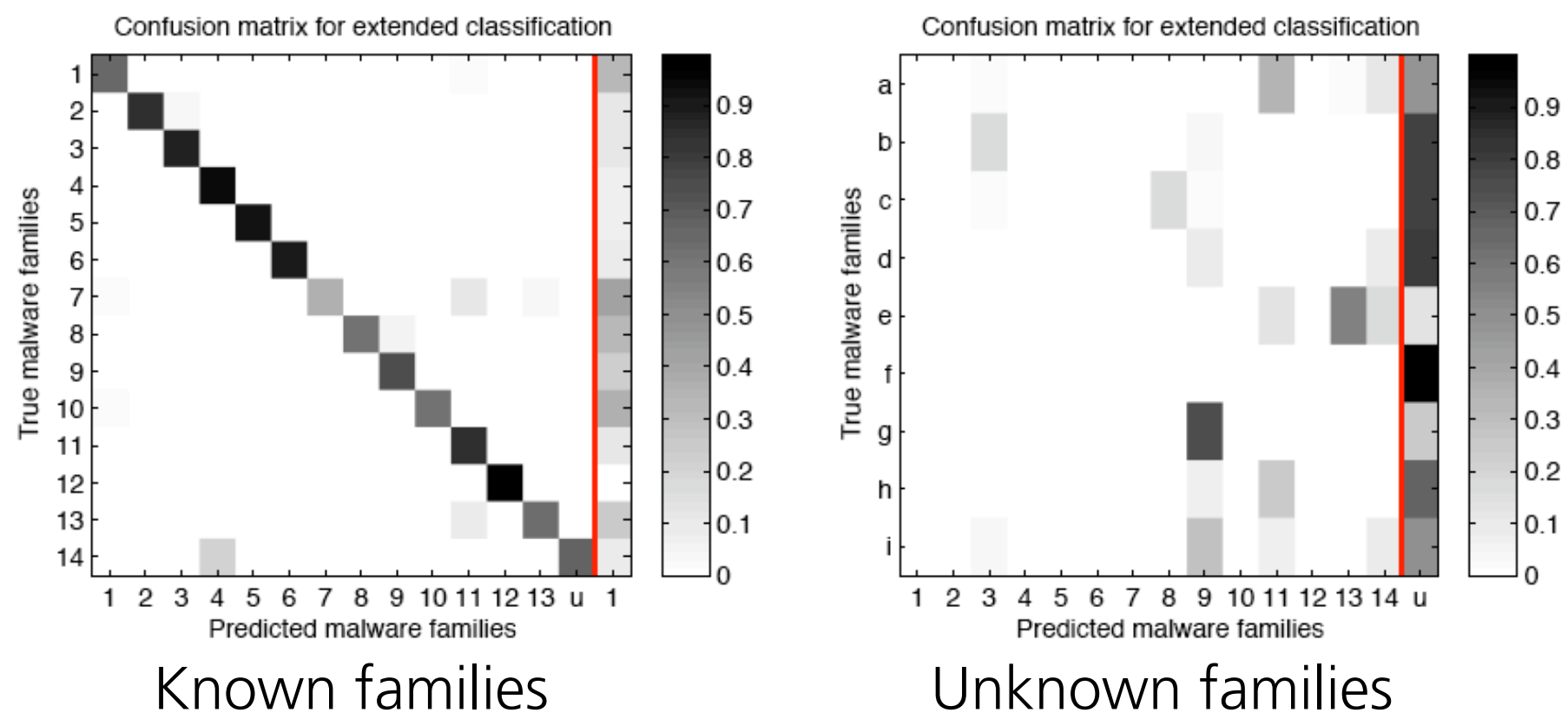

- Reliable rejection of unknown behavior, yet accuracy decreases from $88 \%$ to $73 \%$ 


\section{Feature space}

- Embedding to high-dimensional vector space

- Each operation spans several dimensions

- >1,000,000 and more dimensions

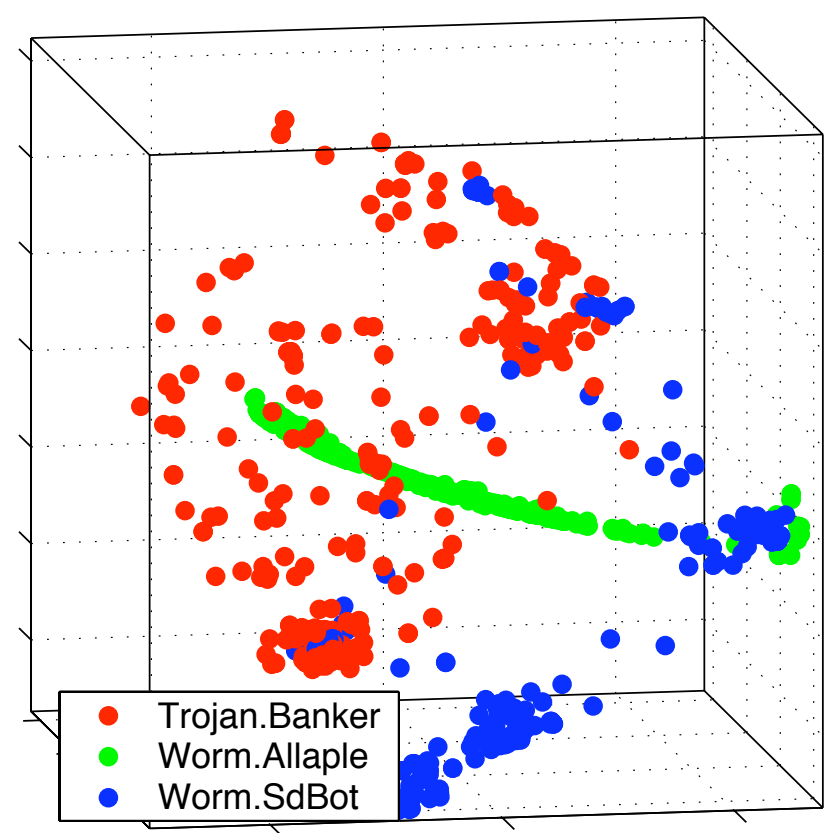

- Visualization using projections (e.g. with PCA) 